\title{
3D-structure of galactic disks
}

\section{Ekaterina Chudakova}

Sternberg Astronomical Institute, Lomonosov Moscow State University, email: artenik@gmail.com

\begin{abstract}
We suggest a new photometric method to estimate thicknesses of galactic disks which is working for the galaxies seen under arbitrary inclination. We have applied our method to 45 galaxies from the open SDSS archive and to 57 galaxies from the Spitzer Survey S4G).
\end{abstract}

Keywords. galaxies: elliptical and lenticular, cD - galaxies: spiral - galaxies: structure

We propose a new photometric method to estimate thickness of a galactic disk seen under arbitrary inclination. We stress two profits of our method: it provides an individual estimate for every galaxy and for the galaxies with orientation different from edge-on it allows to confront its vertical structure to the radial scale. We apply our method to two samples of disk galaxies which radial brightness profiles are already known: to the $r$-band SDSS images of the galaxies which structures are described by Erwin et al. (2008) and Gutiérrez et al. (2011) and to the 3.6- and 4.5- $\mu \mathrm{m}$ images collected by the Spitzer telescope during the survey $\mathrm{S} 4 \mathrm{G}$. The radial structures of the S4G sample are described by Muñoz-Mateos et al. (2013). In the first sample we have obtained very different thickness distributions for the purely exponential-profile galactic disks (Type I) and for the inner disks of antitruncated stellar disks (Type III) (Chudakova \& Sil'chenko 2014).

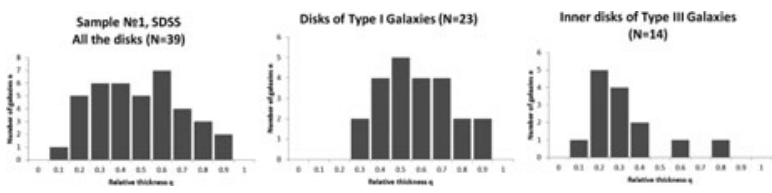

Figure 1. Sample no.1: Distributions of the relative thicknesses $q$.

In the second sample for most galaxies we have two images: in the 3.6- and in the 4.5$\mu \mathrm{m}$ bands. Good agreement between relative thicknesses found in the $3.6-$ and $4.5-\mu \mathrm{m}$ bands shows us the stability of our method.
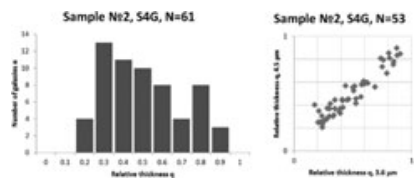

Figure 2. The $q$ distribution and the correlation between the values found in two bands.

\section{References}

Erwin, P., Pohlen, M., \& Beckman, J. E. 2008, AJ, 135, 20

Gutiérrez, L., Erwin, P., Aladro, R., \& Beckman, J. E. 2011, AJ, 142, 145

Muñoz-Mateos, J. C., Sheth, K., Gil de Paz, A., et al. 2013, ApJ, 771, aid 59

Chudakova, E. M. \& Sil'chenko, O. K., 2014, Astron. Rep., 58, 281 